\title{
DOES ENERGY DRINK PROVOKES GAIT MODIFICATIONS IN YOUNG HEALTHY ADULTS?
}

\author{
Martin G. \\ Rosario $^{1+}$ \\ Jason Hogle ${ }^{2}$ \\ Brooks Williams ${ }^{s}$
}

\author{
1,2,s Texas Woman's University, Physical Therapy Program, Dallas Campus; \\ Texas, USA. \\ 'Email:Mrosario1@two.eduTel:214-689-7712 \\ ${ }^{2}$ Email: jhogle@twu.edu \\ ${ }^{3}$ Email: Bwilliams59@twou.edu
}

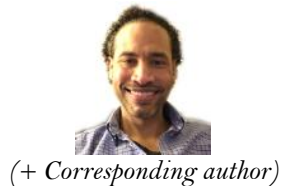

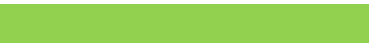

Article History

Received: 10 August 2021 Revised: 13 September 202 Accepted: 6 October 2021

Published: 29 October 2021

\section{Keywords}

Energy drink ingredients

Motor control

Walking

Gait deviations

Red bull

Rockstar

Bang.

\begin{abstract}
Energy drinks have become increasingly popular among young adults and athletes in the last few years. Despite their popularity, little research has substantiated the claims of the positive effects on physical performance of popular energy drinks. Most current research focuses on caffeine alone, but does not often look at how mixing it with other psychoactive substances might alter its effects. Purpose: This study aimed to determine the alterations of gait tasks after consumption of three popular energy drinks on young healthy adults. Methods: Fifteen women and five men were recruited, screened, and signed informed consent to participate in this study. All participants were healthy young adults with no apparent comorbidities that might have been impacted by the consumption of energy drinks. Motion analysis was conducted using Movement Lab TM sensors placed at key anatomical points. Subjects were asked for two bouts of gait tasks pre-energy drink consumption, and then repeat the same tasks after energy drink consumption. Results: There were no significant modifications in gait, however, observable trends were detected in postural gait parameters following the consumption of a Rockstar energy drink. Conclusion: We infer that the trends observed could be attributed to the specific key ingredients used in Rockstar and might have caused gait deviations post consumption. Further studies should focus on Rockstar alone and its specific key ingredients of caffeine, guarana, ginseng, and milk thistle to determine their influence on gait deviations.
\end{abstract}

Contribution/Originality: The paper's primary contribution is finding gait adaptations to diverse energy drinks or energy drinks ingredients in healthy young adults. Particularly, this research identified trends towards gait variations that could be associated to the different ingredients in energy drinks and the interactions of these ingredients with caffeine.

\section{INTRODUCTION}

Energy drinks (E drinks) since showing up on the market in the 1960s have gained immense popularity (1). By 2006, there were around 500 brands of energy drinks available worldwide (1). Presently, they are advertised toward adolescents and young adults with claims of positive effects on performance and mental focus upon consumption (1). They can boast these claims because of their extensive and complex ingredient lists, which are often unresearched and minimally investigated. The primary active element in practically every E drink is caffeine, the most exploited psychoactive drug in the world (2). Besides caffeine, a plethora of diverse supplements are commonly combined with E drinks, including guarana, taurine, cocoa, riboflavin, pyridoxine, nicotinamide, and other derivatives of herbs (2). Although the ingredients in $\mathrm{E}$ drinks are numerous, the focus of most scientific studies is caffeine. Regardless of the 
claims of the diverse $\mathrm{E}$ drink brands and corporations, these ingredients and their effects on human physiology are yet to be determined, equating to a gap in evidence-based literature.

The available research suggests that caffeine is beneficial for enhancing the performance. Grgic, Trexler, Lazinica, and Pedisic (2018) discovered that caffeine is effective as an ergogenic aid for muscle strength and power (5). Other studies showed that caffeine had a modest positive effect on endurance performance; however, further research is needed before being prescribed to athletes (8). In comparison, others indicate that caffeine enhances performance endurance because of the combined effects of increased lipolysis and positive influence on nerve impulse transmission (14). However, even though caffeine has many detailed benefits in improving performance, there is one evident break in the available knowledge: What are the effects of caffeine when incorporated with various stimulants? Explicitly, how do the different and fluctuating ingredients in E drinks impact the overall effect of caffeine and, therefore, various physiological systems?

Recent examinations of $\mathrm{E}$ drinks have attempted to delve into the detrimental and beneficial effects of its usage. Among the adverse effects of $\mathrm{E}$ drinks, healthy young adults exhibited an increase in blood pressure (BP), platelet aggregation, and deterioration of endothelial function one hour after ingesting an energy drink (9) as well as a significant increase in heart rate, which enhances the risk of dangerous cardiovascular events (12). What is concerning is the varying information related to E drinks, such as that illustrated in a systematic review. The report pointed out that the impact of $\mathrm{E}$ drinks on an average person's health is insufficient and unfounded; however, the same examination noticed that E drinks might help improve aerobic endurance (13). Sugar-free Red Bull shortened simple reaction time, and enhanced pre-movement facilitation(4); however, other studies recommend limiting consumption not avoiding $\mathrm{E}$ drinks for athletes even after recognizing the fact that drinks could lead to dehydration and increased BP (7).

There are claims that $\mathrm{E}$ drinks are harmless unless they are over consumed, but there are still concerns over the surfeit of ingredients apart from the caffeine in energy drinks (6) that could disturb diverse systems. Guarana, for instance, contains theobromine and theophylline, which are mild central nervous system (CNS) stimulants that have been proven to energize more than caffeine alone in non-human models with a CNS comparable to those of mammals (3). The influence of caffeine or E drinks on gait stability has yet to be examined. To date, three studies have investigated these effects. In Rosario, Collazo, Mateo, Gonzalez-Sola, and Bayron (2017) discovered that 16 ounces of an energy drink did not significantly impair postural control in healthy young adults. However, the authors noted a postural instability trend after consuming the drinks compared to the caffeine group (10). Furthermore, Rosario, Clare, Lauren, and Ashley (2021) observed altered neuromuscular time activity in leg musculature during a balance test after 16 ounces E Drink. These results differ from those of a study by McNerney, Coad, and Burkard (2014) that found caffeine ingestion did not influence the sensory organization test (11). Due to these opposing results, more research needs to be conducted to verify the effects of energy drinks on postural control, sensory organization, and memory. Considering all those referred to above, the query this investigation seeks to explain is, do energy drinks modify gait and balance? We found that energy drinks might create gait and balance variation. This examination will add to the gap related to the influence of energy drinks on memory and muscle activity/fatigue that could disturb learning and health.

\section{METHODS}

We recruited 20 healthy adults ( 15 women and $5 \mathrm{men}$ ). This recruitment was from a convenience sample. The average participant age was $24.57 \pm 3.47$. All participants, except one, were TWU students currently enrolled in either the PT or OT programs. Exclusion criteria included a history of diabetes, heart conditions, and injuries or surgeries of the arm, elbow, or leg six months prior to the study. After signing the informed consent forms, the participants' height, weight, blood pressure, and pulse were measured. Once baseline vitals were collected, a gait protocol was initiated. To collect data for motion analysis during gait, movement lab sensors were utilized. In this 
experiment, a sensor was placed on each of five key anatomical points over the lumbar spine, bilateral arms, and bilateral legs. All of these sensors collected sway velocity, direction, and distance during all balance and gait tasks. These sensors provide insight into body movements in the sagittal and frontal planes. Following the above, the participants started the gait protocol of the experiment. The participants were asked to stand behind a green line on the floor and were instructed to start walking once they heard a beep. Once the participant started walking, they were told to walk $7 \mathrm{~m}$ until they reached a cone. The instructions were to walk around the cone and then walk back to the green line and then repeat that until the last beep sounded after a duration of 30 seconds. When they heard the last beep, they were instructed to continue walking back to the green line start. The same conditions were repeated for the second walk. After performing the gait tasks, the participants drank one of three energy drinks, red bulls, Rockstar, or Bang. Bang was the only drink sugar-free and were all the drinks were the most popular flavors of their respective brands. The subjects were given 10 minutes to finish consuming the energy drink. Once the energy drink was consumed, they were checked with a pulse monitor on the middle finger of their dominant hand. Once their pulse decreased or increased by 10 beats per minute from their baseline measurement at the beginning of the study, we began the second round of testing, post E drinks. After drinking the energy drink, participant then moved on to the gait protocol. Upon completion of the test, the participants were thanked and given a $\$ 15$ Amazon gift card for contributing in the study.

\section{RESULTS}

Table 1 contains the demographics of the study, which included age, sex, BMI, height, and weight for each energy drink group. Eighteen of the 20 participants were students from the TWU Dallas physical therapy program, 1 was from the TWU Dallas occupational therapy program, and the last participant had no affiliation with the school but was similar in all other characteristics to the previous 19 participants. The energy drink groups observed in this study are Redbull, Rockstar, and Bang. Overall, females made up the largest population in this study: 15 females compared to five males. There were two males and five females in the Redbull group, one male and six females in the Bang group, and two males and four females in the Rockstar group. The average age of the Redbull group was $24.88 \pm 5.13$, the average body mass index (BMI) was 23.46, and all were right-leg dominant. The average age of the Bang group was $24.67 \pm 1.86$, average BMI was 22.5, and 5 participants were right leg dominant while 1 was left leg dominant. The average age of the Rockstar group was $23.83 \pm 2.32$, the average BMI was 25.0, and all of these participants were right-leg dominant. Blood pressure (BP) and heart rate (HR) were measured for every participant before additional data were collected. No participants were found to be hypertensive; however, the Bang group had a higher starting HR of $77.42 \pm 10.77$. Oxygen saturation was additionally collected from each participant and was found to be within the normal range for each group.

Table-1. Demographic data of all participants.

\begin{tabular}{|c|c|c|c|}
\hline Characteristics & $\begin{array}{c}\text { Participant Data } \\
\text { REDBULL }\end{array}$ & $\begin{array}{c}\text { Participant Data } \\
\text { BANG }\end{array}$ & $\begin{array}{c}\text { Participant Data ROCK } \\
\text { STAR }\end{array}$ \\
\hline Age & $24.88+/-5.13$ & $24.67 \pm 1.86$ & $23.83 \pm 2.32$ \\
\hline Gender & Male $=2$ Female $=5$ & Male $=1$ Female $=6$ & Male $=2$ Female $=4$ \\
\hline Height (in) & $65.85+/-3.27$ & $64.43+/-3.64$ & $66.66+/-1.97$ \\
\hline Weight (lb) & $145.39+/-23.86$ & $130.91+/-23.13$ & $154.97+/-23.79$ \\
\hline $\mathrm{BMI}\left(\mathrm{kg} / \mathrm{m}^{\wedge} \mathcal{Q}\right)$ & $23.46+/-2.87$ & 22.5 & 25.0 \\
\hline Heart Rate (bpm) & $69.82+/-11.58$ & $77.42+/-10.77$ & $72.5+/-9.22$ \\
\hline Systolic BP (mmHg) & $118.82+/-15.75$ & $112.71+/-8.94$ & $115.83+/-11.16$ \\
\hline Diastolic BP (mmHg) & $76.27+/-9.09$ & $73.86+/-6.09$ & $73.86+/-4.88$ \\
\hline Sat $\mathrm{O} 2(\%)$ & $97.97+/-2.23$ & $98.42+/-0.534$ & $98.33+/-0.52$ \\
\hline Leg Dominance & $\mathrm{R}=7$ & $\begin{array}{l}\mathrm{R}=5 \\
\mathrm{~L}=1\end{array}$ & $\mathrm{R}=6$ \\
\hline
\end{tabular}


Table-2. Comparisons of gait variables among tasks. Results of repeated measure ANOVA performed comparing single and dual. Significance level set at $\mathrm{p} \leq 0.01$.

\begin{tabular}{|c|c|c|c|c|c|c|c|c|c|}
\hline & $\begin{array}{l}\text { PRE } \\
\text { Means and SD }\end{array}$ & $\begin{array}{l}\text { REDBULL } \\
\text { Means and SD }\end{array}$ & P-value & $\begin{array}{l}\text { PRE } \\
\text { Means and SD }\end{array}$ & $\begin{array}{l}\text { BANG } \\
\text { Means and SD }\end{array}$ & $\begin{array}{l}\mathrm{P}- \\
\text { value }\end{array}$ & $\begin{array}{l}\text { PRE } \\
\text { Means and SD }\end{array}$ & $\begin{array}{l}\text { ROCK STAR } \\
\text { Means a d SD }\end{array}$ & P-value \\
\hline Cadence L (steps/min) & $110.20 \pm 6.59$ & $105.92 \pm 8.99$ & 0.30 & $112.24 \pm 6.10$ & $114.70 \pm 6.81$ & 0.35 & $108.65 \pm 5.47$ & $109.12 \pm 5.10$ & 0.83 \\
\hline Cadence R (steps/min) & $110.08 \pm 6.58$ & $105.92 \pm 8.98$ & 0.95 & $112.17 \pm 5.94$ & $114.84 \pm 6.68$ & 0.30 & $108.70 \pm 5.25$ & $109.18 \pm 5.02$ & 0.82 \\
\hline Cadence Asymmetry (\% diff) & $-0.13 \pm 0.43$ & $-0.00 \pm 0.34$ & 0.87 & $-0.06 \pm 0.30$ & $0.13 \pm 0.29$ & 0.12 & $0.06 \pm 0.30$ & $0.05 \pm 0.21$ & 0.94 \\
\hline Double Support L (\%GCT) & $19.26 \pm 2.42$ & $20.98 \pm 3.30$ & 0.65 & $18.27 \pm 2.93$ & $17.62 \pm 2.64$ & 0.57 & $18.14 \pm 1.06$ & $17.96 \pm 1.41$ & 0.76 \\
\hline Double Support R (\%GCT) & $19.38 \pm 2.38$ & $21.02 \pm 3.29$ & 0.68 & $18.39 \pm 2.93$ & $17.70 \pm 2.63$ & 0.55 & $18.12 \pm 0.99$ & $18.04 \pm 1.39$ & 0.87 \\
\hline $\begin{array}{l}\text { Double } \quad \text { Support } \quad \text { Asymmetry } \\
(\% \text { Diff })\end{array}$ & $0.64 \pm 0.95$ & $0.24 \pm 0.62$ & 0.54 & $0.65 \pm 0.67$ & $0.53 \pm 1.53$ & 0.79 & $-0.12 \pm 0.88$ & $0.46 \pm 0.45$ & 0.05 \\
\hline Elevation at Midswing L (cm) & $1.00 \pm 0.52$ & $0.92 \pm 0.48$ & 0.97 & $0.89 \pm 0.53$ & $0.95 \pm 0.49$ & 0.78 & $0.85 \pm 0.50$ & $0.81 \pm 0.58$ & 0.84 \\
\hline Elevation at Midswing R (cm) & $0.86 \pm 0.51$ & $0.89 \pm 0.45$ & 0.63 & $0.82 \pm 0.30$ & $0.93 \pm 0.44$ & 0.45 & $0.64 \pm 0.56$ & $0.62 \pm 0.64$ & 0.95 \\
\hline $\begin{array}{l}\text { Elevation } \quad \text { at } \\
\text { Asymmetry (\%Diff) }\end{array}$ & $-12.67 \pm 61.09$ & $2.30 \pm 52.40$ & 0.66 & $12.40 \pm 53.89$ & $7.13 \pm 43.72$ & 0.80 & $-44.21 \pm 46.54$ & $-19.19 \pm 81.63$ & 0.36 \\
\hline Gait Cycle Duration L (s) & $1.09 \pm 0.07$ & $1.14 \pm 0.10$ & 0.91 & $1.07 \pm 0.06$ & $1.05 \pm 0.06$ & 0.32 & $1.11 \pm 0.06$ & $1.10 \pm 0.05$ & 0.85 \\
\hline Gait Cycle Duration R (s) & $1.10 \pm 0.07$ & $1.14 \pm 0.10$ & 0.90 & $1.07 \pm 0.06$ & $1.05 \pm 0.06$ & 0.29 & $1.11 \pm 0.06$ & $1.10 \pm 0.05$ & 0.87 \\
\hline $\begin{array}{l}\text { Gait Cycle Duration Asymmetry } \\
\text { (\%Diff) }\end{array}$ & $0.12 \pm 0.44$ & -0.01 & 0.92 & $0.04 \pm 0.32$ & $-0.17 \pm 0.22$ & 0.07 & $-0.10 \pm 0.30$ & $-0.02 \pm 0.22$ & 0.47 \\
\hline Gait Speed L (m/s) & $1.16 \pm 0.12$ & $1.08 \pm 0.13$ & 0.99 & $1.14 \pm 0.09$ & $1.21 \pm 0.15$ & 0.17 & $1.15 \pm 0.18$ & $1.15 \pm 0.19$ & 0.97 \\
\hline Gait Speed R (m/s) & $1.14 \pm 0.11$ & $1.07 \pm 0.13$ & 0.95 & $1.13 \pm 0.09$ & $1.19 \pm 0.15$ & 0.19 & $1.15 \pm 0.18$ & $1.15 \pm 0.19$ & 0.99 \\
\hline Gait Speed Asymmetry (\%Diff) & $-1.17 \pm 1.90$ & $-0.96 \pm 1.98$ & 0.73 & $-0.84 \pm 1.29$ & $-1.38 \pm 1.46$ & 0.33 & $0.06 \pm 1.33$ & $-0.24 \pm 1.42$ & 0.59 \\
\hline Lateral Step Variability L (cm) & $3.83 \pm 0.90$ & $4.05 \pm 1.28$ & 0.61 & $4.00 \pm 1.34$ & $4.45 \pm 1.42$ & 0.42 & $4.15 \pm 1.24$ & $4.37 \pm 1.10$ & 0.64 \\
\hline
\end{tabular}


Journal of Sports Research, 202 1, 8(2): 82-92

\begin{tabular}{|c|c|c|c|c|c|c|c|c|c|}
\hline Lateral Step Variability R (cm) & $3.60 \pm 1.07$ & $3.57 \pm 1.17$ & 0.62 & $3.64 \pm 1.2$ & $3.63 \pm 1.4$ & 0.73 & $4.06 \pm 0.88$ & $4.11 \pm 1.14$ & 0.91 \\
\hline $\begin{array}{lcl}\text { Lateral } & \text { Step } & \text { Variability } \\
\text { Asymmetry (\%Diff) } & \end{array}$ & $-7.78 \pm 22.57$ & $-12.55 \pm 28.18$ & ??? & $3.06 \pm 30.89$ & $-1.74 \pm 16.59$ & 0.64 & $-0.21 \pm 35.28$ & $-7.02 \pm 19.26$ & 0.88 \\
\hline Circumduction L (cm) & $3.66 \pm 1.20$ & $3.87 \pm 1.48$ & 0.78 & $3.02 \pm 1.38$ & $3.26 \pm 1.45$ & 0.67 & $3.35 \pm 1.31$ & $3.15 \pm 1.48$ & 0.72 \\
\hline Circumduction R (cm) & $3.57 \pm 1.16$ & $3.74 \pm 1.43$ & 0.80 & $3.30 \pm 1.32$ & $3.31 \pm 1.42$ & 0.99 & $3.35 \pm 1.43$ & $3.26 \pm 1.51$ & 0.89 \\
\hline $\begin{array}{l}\text { Circumduction } \\
\text { (\%Diff) }\end{array}$ & $-3.87 \pm 25.08$ & $-3.90 \pm 30.12$ & 0.80 & $16.56 \pm 35.65$ & $7.19 \pm 40.35$ & 0.54 & $-2.78 \pm 8.50$ & $7.49 \pm 19.09$ & 0.10 \\
\hline Lower Limb-N (\#) & $15.96 \pm 1.97$ & $15.69 \pm 2.03$ & 0.85 & $15.57 \pm 2.34$ & $14.64 \pm 3.20$ & 0.41 & $16.9 \pm 1.7$ & $15 . \pm 2.0$ & 0.95 \\
\hline Foot Strike Angle L (degrees) & $26.98 \pm 4.49$ & $26.80 \pm 4.07$ & 0.76 & $25.03 \pm 3.13$ & $25.98 \pm 2.75$ & 0.43 & $27.76 \pm 5.63$ & $26.84 \pm 6.25$ & 0.70 \\
\hline Foot Strike Angle R (degrees) & $26.43 \pm 3.33$ & $26.43 \pm 3.19$ & 0.15 & $23.93 \pm 2.45$ & $25.40 \pm 1.87$ & 0.11 & $26.71 \pm 6.59$ & $26.06 \pm 6.62$ & 0.81 \\
\hline $\begin{array}{l}\text { Foot Strike Angle Asymmetry } \\
\text { (\%Diff) }\end{array}$ & $-1.84 \pm 12.23$ & $-1.18 \pm 10.82$ & 0.13 & $-4.18 \pm 4.87$ & $-1.97 \pm 7.88$ & 0.40 & $-2.20 \pm 4.3$ & $-2.97 \pm 7.7$ & 0.40 \\
\hline Toe Off Angle L (degrees) & $38.03 \pm 3.13$ & $36.70 \pm 3.81$ & 0.53 & $39.73 \pm 2.75$ & $39.52 \pm 3.18$ & 0.86 & $38.07 \pm 3.59$ & $38.04 \pm 2.80$ & 0.98 \\
\hline Toe Off Angle R (degrees) & $37.91 \pm 3.43$ & $36.65 \pm 3.32$ & 0.91 & $39.97 \pm 1.52$ & $40.54 \pm 1.70$ & 0.39 & $39.55 \pm 3.99$ & $38.47 \pm 3.15$ & 0.46 \\
\hline Toe Off Angle Asymmetry (\%Diff) & $-0.56 \pm 8.12$ & $0.04 \pm 10.08$ & 0.40 & $0.80 \pm 6.32$ & $2.82 \pm 6.80$ & 0.45 & $3.70 \pm 5.43$ & $1.03 \pm 3.92$ & 0.17 \\
\hline Single Limb Support L (\%GCT) & $40.39 \pm 1.30$ & $39.64 \pm 1.75$ & 0.96 & $40.87 \pm 1.53$ & $41.22 \pm 1.60$ & 0.58 & $41.18 \pm 0.83$ & $41.35 \pm 0.66$ & 0.57 \\
\hline Single Limb Support R (\%GCT) & $40.30 \pm 1.30$ & $39.38 \pm 1.77$ & 0.40 & $40.84 \pm 1.44$ & $41.22 \pm 1.07$ & 0.47 & $40.70 \pm 0.43$ & $40.71 \pm 0.83$ & 0.98 \\
\hline $\begin{array}{l}\text { Single Limb Support Asymmetry } \\
\text { (\%Diff) }\end{array}$ & $-0.24 \pm 2.57$ & $-0.65 \pm 2.97$ & 0.47 & $-0.06 \pm 1.76$ & $0.03 \pm 1.86$ & 0.91 & $-1.17 \pm 2.11$ & $-1.58 \pm 1.12$ & 0.54 \\
\hline Stance L (\%GCT) & $59.65 \pm 1.33$ & $60.61 \pm 1.74$ & 0.41 & $59.14 \pm 1.48$ & $58.84 \pm 1.09$ & 0.58 & $59.33 \pm 0.47$ & $59.31 \pm 0.81$ & 0.95 \\
\hline Stance R (\%GCT) & $59.68 \pm 1.31$ & $60.41 \pm 1.73$ & 0.96 & $59.22 \pm 1.57$ & $58.92 \pm 1.61$ & 0.64 & $58.82 \pm 0.83$ & $58.75 \pm 0.62$ & 0.81 \\
\hline Stance Asymmetry (\%Diff) & $0.05 \pm 1.77$ & $-0.35 \pm 1.87$ & 0.40 & $0.14 \pm 1.11$ & $0.13 \pm 1.22$ & 0.97 & $-0.86 \pm 1.44$ & $-0.94 \pm 0.76$ & 0.85 \\
\hline Step Duration L (s) & $0.55 \pm 0.04$ & $0.57 \pm 0.05$ & 0.93 & $0.54 \pm 0.03$ & $0.53 \pm 0.04$ & 0.48 & $0.55 \pm 0.02$ & $0.55 \pm 0.03$ & 0.64 \\
\hline
\end{tabular}


Journal of Sports Research, 202 1, 8(2): 82-92

\begin{tabular}{|c|c|c|c|c|c|c|c|c|c|}
\hline Step Duration R (s) & $0.55 \pm 0.03$ & $0.57 \pm 0.05$ & 0.86 & $0.54 \pm 0.03$ & $0.52 \pm 0.03$ & 0.29 & $0.56 \pm 0.03$ & $0.55 \pm 0.03$ & 0.92 \\
\hline Step Duration Asymmetry (\%Diff) & $-0.56 \pm 2.81$ & $-0.18 \pm 2.62$ & 0.84 & $0.07 \pm 2.58$ & $-0.28 \pm 3.08$ & 0.76 & $0.88 \pm 3.10$ & $1.50 \pm 2.33$ & 0.58 \\
\hline Stride Length L (m) & $1.26 \pm 0.10$ & $1.22 \pm 0.11$ & 0.97 & $1.22 \pm 0.07$ & $1.26 \pm 0.11$ & 0.23 & $1.26 \pm 0.15$ & $1.26 \pm 0.16$ & 0.97 \\
\hline Stride Length R (m) & $1.24 \pm 0.10$ & $1.21 \pm 0.10$ & 0.99 & $1.20 \pm 0.06$ & $1.24 \pm 0.10$ & 0.28 & $1.26 \pm 0.15$ & $1.25 \pm 0.16$ & 0.90 \\
\hline Stride Length Asymmetry (\%Diff) & $-1.09 \pm 2.05$ & $-0.92 \pm 1.78$ & 0.71 & $-0.89 \pm 1.30$ & $-1.50 \pm 1.49$ & 0.29 & $-0.02 \pm 1.34$ & $-0.33 \pm 1.40$ & 0.58 \\
\hline Swing L (\%GCT) & $40.35 \pm 1.33$ & $39.38 \pm 1.74$ & 0.40 & $40.86 \pm 1.48$ & $41.16 \pm 1.09$ & 0.58 & $40.67 \pm 0.47$ & $40.69 \pm 0.81$ & 0.96 \\
\hline Swing R (\%GCT) & $40.32 \pm 1.31$ & $39.59 \pm 1.73$ & 0.96 & $40.78 \pm 1.57$ & $41.08 \pm 1.61$ & 0.64 & $41.18 \pm 0.83$ & $41.25 \pm 0.62$ & 0.81 \\
\hline Swing Asymmetry (\%Diff) & $-0.09 \pm 2.66$ & $0.53 \pm 2.88$ & 0.39 & $-0.22 \pm 1.68$ & $-0.25 \pm 1.78$ & 0.96 & $1.22 \pm 2.06$ & $1.38 \pm 1.10$ & 0.80 \\
\hline $\begin{array}{l}\text { Terminal Double Support L } \\
(\% \text { GCT })\end{array}$ & $9.84 \pm 1.39$ & $10.67 \pm 1.93$ & 0.58 & $9.15 \pm 1.34$ & $8.83 \pm 1.45$ & 0.58 & $9.04 \pm 0.67$ & $8.91 \pm 0.95$ & 0.69 \\
\hline $\begin{array}{l}\text { Terminal Double Support } \mathrm{R} \\
(\% \mathrm{GCT})\end{array}$ & $9.56 \pm 1.22$ & $10.36 \pm 1.51$ & 0.99 & $9.26 \pm 1.65$ & $8.88 \pm 1.31$ & 0.53 & $9.07 \pm 0.65$ & $9.13 \pm 0.84$ & 0.86 \\
\hline $\begin{array}{l}\text { Terminal Double } \quad \text { Support } \\
\text { Asymmetry (\%Diff) }\end{array}$ & $-2.68 \pm 12.12$ & $-2.28 \pm 10.85$ & 0.45 & $0.55 \pm 10.39$ & $0.78 \pm 11.85$ & 0.96 & $0.26 \pm 10.06$ & $2.50 \pm 12.36$ & 0.63 \\
\hline Toe Out Angle L (degrees) & $5.12 \pm 5.48$ & $6.26 \pm 6.73$ & 0.97 & $1.30 \pm 10.37$ & $1.20 \pm 12.43$ & 0.98 & $1.84 \pm 6.55$ & $3.58 \pm 5.90$ & 0.49 \\
\hline Toe Out Angle R (degrees) & $11.06 \pm 6.36$ & $11.16 \pm 6.50$ & 0.14 & $13.28 \pm 5.08$ & $12.44 \pm 3.78$ & 0.66 & $7.98 \pm 4.49$ & $8.68 \pm 2.53$ & 0.63 \\
\hline $\begin{array}{l}\text { Lumbar Range of Motion } \\
\text { (Coronal) }\end{array}$ & $7.34 \pm 1.99$ & $7.39 \pm 1.71$ & 0.42 & $5.66 \pm 2.49$ & $6.59 \pm 2.57$ & 0.81 & $7.48 \pm 1.48$ & $6.39 \pm 1.37$ & 0.06 \\
\hline $\begin{array}{l}\text { Lumbar Range of Motion } \\
\text { (Sagittal) }\end{array}$ & $5.03 \pm 1.49$ & $5.17 \pm 1.75$ & 0.83 & $4.80 \pm 0.77$ & $5.24 \pm 0.61$ & 0.13 & $4.67 \pm 0.66$ & $5.07 \pm 1.08$ & 0.25 \\
\hline $\begin{array}{l}\text { Lumbar Range of Motion } \\
\text { (Transverse) (degrees) }\end{array}$ & $10.10 \pm 3.56$ & $10.50 \pm 3.51$ & 0.82 & $7.84 \pm 1.79$ & $8.59 \pm 2.02$ & 0.36 & $8.68 \pm 2.32$ & $8.42 \pm 2.43$ & 0.77 \\
\hline $\begin{array}{l}\text { Trunk Range of Motion (Coronal) } \\
\text { (degrees) }\end{array}$ & $5.30 \pm 2.16$ & $6.22 \pm 2.21$ & 0.68 & $4.45 \pm 0.99$ & $4.74 \pm 0.95$ & 0.47 & $4.37 \pm 1.23$ & $5.04 \pm 1.53$ & 0.21 \\
\hline
\end{tabular}


Journal of Sports Research, 2021, 8(2): 82-92

\begin{tabular}{|c|c|c|c|c|c|c|c|c|c|}
\hline Arm Swing Velocity L (degrees/s) & $237.85 \pm 62.43$ & $267.34 \pm 82.17$ & 0.98 & $245.63 \pm 62.59$ & $275.94 \pm 72.28$ & 0.27 & $216.62 \pm 71.32$ & $231.41 \pm 42.10$ & 0.51 \\
\hline Arm Swing Velocity R (degrees/s) & $207.53 \pm 59.21$ & $226.77 \pm 85.52$ & 0.70 & $199.88 \pm 67.27$ & $209.58 \pm 67.21$ & 0.72 & $197.04 \pm 75.98$ & $201.47 \pm 71.24$ & 0.88 \\
\hline Arm Range of Motion L (degrees) & $57.78 \pm 17.33$ & $63.41 \pm 19.20$ & 0.86 & $56.77 \pm 17.17$ & $65.67 \pm 15.89$ & 0.20 & $46.36 \pm 8.64$ & $52.03 \pm 4.66$ & 0.05 \\
\hline Arm Range of Motion R (degrees) & $54.05 \pm 15.36$ & $56.03 \pm 18.72$ & 0.36 & $50.96 \pm 20.09$ & $51.73 \pm 21.70$ & 0.93 & $48.05 \pm 15.17$ & $47.86 \pm 16.89$ & 0.85 \\
\hline
\end{tabular}

Table-3. Comparisons of Anticipatory Postural Adjustments among tasks. Results of repeated measure ANOVA performed comparing single and dual. Significance level set at $\mathrm{p} \leq 0.01$.

\begin{tabular}{|c|c|c|c|c|c|c|c|c|c|}
\hline & $\begin{array}{c}\text { PRE Drink } \\
\text { Means and SD }\end{array}$ & $\begin{array}{c}\text { REBULL } \\
\text { Means and SD }\end{array}$ & $\begin{array}{c}P- \\
\text { value }\end{array}$ & $\begin{array}{c}\text { PRE Drink } \\
\text { Means and SD }\end{array}$ & $\begin{array}{c}\text { BANG } \\
\text { Means and SD }\end{array}$ & $\begin{array}{c}\mathrm{P}- \\
\text { value }\end{array}$ & $\begin{array}{l}\text { PRE Drink } \\
\text { Means and } \\
\text { SD }\end{array}$ & $\begin{array}{c}\text { ROCK STAR } \\
\text { Means and } \\
\text { SD }\end{array}$ & $\begin{array}{c}\mathrm{P}- \\
\text { value }\end{array}$ \\
\hline $\begin{array}{l}\text { Anticipatory Postural Adjustment - APA } \\
\text { Duration (s) }\end{array}$ & $0.39 \pm 0.26$ & $0.49 \pm 0.31$ & 0.50 & $0.35 \pm 0.23$ & $0.30 \pm 0.07$ & 0.49 & $0.33 \pm 0.13$ & $0.45 \pm 0.42$ & 0.45 \\
\hline $\begin{array}{l}\text { Anticipatory Postural Adjustment - First } \\
\text { Step Duration (s) }\end{array}$ & $0.51 \pm 0.06$ & $0.54 \pm 0.07$ & 0.78 & $0.51 \pm 0.03$ & $0.50 \pm 0.04$ & 0.61 & $0.52 \pm 0.03$ & $0.50 \pm 0.03$ & 0.32 \\
\hline $\begin{array}{l}\text { Anticipatory Postural Adjustment - First } \\
\text { Step Range of Motion (degrees) }\end{array}$ & $32.20 \pm 8.56$ & $37.84 \pm 9.53$ & 0.71 & $31.44 \pm 6.30$ & $35.65 \pm 19.16$ & 0.48 & $36.74 \pm 11.62$ & $42.14 \pm 11.68$ & 0.39 \\
\hline $\begin{array}{l}\text { Anticipatory Postural Adjustment - } \\
\text { Forward APA Peak }(\mathrm{m} / \mathrm{s} 2)\end{array}$ & $0.50 \pm 0.24$ & $0.59 \pm 0.31$ & 0.60 & $0.42 \pm 0.18$ & $0.52 \pm 0.37$ & 0.42 & $0.51 \pm 0.34$ & $0.87 \pm 0.36$ & 0.07 \\
\hline $\begin{array}{l}\text { Anticipatory Postural Adjustment - } \\
\text { Lateral APA Peak }(\mathrm{m} / \mathrm{s} 2)\end{array}$ & $0.55 \pm 0.23$ & $0.50 \pm 0.19$ & 0.81 & $0.48 \pm 0.23$ & $0.44 \pm 0.14$ & 0.65 & $0.41 \pm 0.21$ & $0.37 \pm 0.15$ & 0.70 \\
\hline
\end{tabular}


Table-4. Comparisons of Posture and Turns among tasks. Results of repeated measure ANOVA performed comparing single and dual. Significance level set at $\mathrm{p} \leq 0.01$.

\begin{tabular}{|c|c|c|c|c|c|c|c|c|c|}
\hline & $\begin{array}{l}\text { Single Tasks } \\
\text { Means and SD }\end{array}$ & $\begin{array}{c}\text { Dual Tasks } \\
\text { Means and SD }\end{array}$ & $\begin{array}{c}\mathrm{P}- \\
\text { value }\end{array}$ & $\begin{array}{c}\text { PRE Drink } \\
\text { Means and SD }\end{array}$ & $\begin{array}{c}\text { BANG } \\
\text { Means and SD }\end{array}$ & $\begin{array}{c}\mathrm{P}- \\
\text { value }\end{array}$ & $\begin{array}{c}\text { PRE Drink } \\
\text { Means and SD }\end{array}$ & $\begin{array}{l}\text { ROCK STAR } \\
\text { Means and SD }\end{array}$ & $\begin{array}{c}\mathrm{P}- \\
\text { value }\end{array}$ \\
\hline $\begin{array}{l}\text { Postural-Sway-Acc-Sway } \\
\text { Area }(\mathrm{m} 2 / \mathrm{s} 4)\end{array}$ & $17.50 \pm 6.71$ & $17.53 \pm 6.03$ & 0.91 & $15.83 \pm 2.96$ & $18.14 \pm 4.91$ & 0.15 & $14.42 \pm 7.85$ & $15.82 \pm 7.69$ & 0.63 \\
\hline $\begin{array}{l}\text { Postural-Sway-Acc-Jerk } \\
\text { (Coronal) (m2/s5) }\end{array}$ & $2601.94 \pm 3242.96$ & $2267.22 \pm 2314.67$ & 0.62 & $2245.89 \pm 1406.30$ & $2955.79 \pm 1747.50$ & 0.26 & $2027.34 \pm 1921.72$ & $2129.00 \pm 1917.92$ & 0.89 \\
\hline $\begin{array}{l}\text { Postural-Sway-Acc-Jerk } \\
\text { (Sagittal) (m2/s5) }\end{array}$ & $6288.39 \pm 2201.43$ & $6703.63 \pm 2480.74$ & 0.89 & $5542.05 \pm 1337.59$ & $6052.49 \pm 1408.44$ & 0.35 & $5210.09 \pm 2146.45$ & $5237.16 \pm 2121.80$ & 0.97 \\
\hline $\begin{array}{l}\text { Postural Sway-Acc-Mean } \\
\text { Velocity (Sagittal) }(\mathrm{m} / \mathrm{s})\end{array}$ & $0.52 \pm 0.18$ & $0.54 \pm 0.17$ & 0.66 & $0.49 \pm 0.29$ & $0.57 \pm 0.22$ & 0.81 & $0.38 \pm 0.30$ & $0.49 \pm 0.26$ & 0.32 \\
\hline $\begin{array}{l}\text { Postural Sway-Acc-Mean } \\
\text { Velocity (Coronal) }(\mathrm{m} / \mathrm{s})\end{array}$ & $0.29 \pm 0.10$ & $0.26 \pm 0.10$ & 0.71 & $0.24 \pm 0.06$ & $0.23 \pm 0.05$ & 0.45 & $0.26 \pm 0.11$ & $0.25 \pm 0.07$ & 0.73 \\
\hline $\begin{array}{l}\text { Postural Sway-Acc-Path } \\
\text { Length (Coronal) }(\mathrm{m} / \mathrm{s} 2)\end{array}$ & $222.61 \pm 108.62$ & $209.13 \pm 96.37$ & 0.70 & $215.46 \pm 76.54$ & $248.75 \pm 92.01$ & 0.32 & $193.51 \pm 105.89$ & $201.61 \pm 99.61$ & 0.84 \\
\hline $\begin{array}{l}\text { Postural Sway-Acc-Path } \\
\text { Length (Sagittal) }(\mathrm{m} / \mathrm{s} 2)\end{array}$ & $383.50 \pm 73.06$ & $389.48 \pm 77.74$ & 0.99 & $368.26 \pm 44.82$ & $386.88 \pm 47.01$ & 0.31 & $344.33 \pm 78.38$ & $346.25 \pm 79.19$ & 0.95 \\
\hline Turns-Angle (degrees) & $179.72 \pm 9.46$ & $178.99 \pm 13.69$ & 0.19 & $179.86 \pm 5.74$ & $178.36 \pm 7.07$ & 0.59 & $185.00 \pm 6.33$ & $182.05 \pm 7.50$ & 0.42 \\
\hline Turns-Duration (s) & $2.22 \pm 0.42$ & $2.31 \pm 0.46$ & 0.72 & $2.49 \pm 0.36$ & $2.39 \pm 0.44$ & 0.57 & $2.47 \pm 0.48$ & $2.12 \pm 0.26$ & 0.11 \\
\hline Turns-N (\#) & $2.86 \pm 0.52$ & $2.62 \pm 0.62$ & 0.61 & $2.67 \pm 0.65$ & $3.00 \pm 0.67$ & 0.25 & $2.75 \pm 0.46$ & $2.86 \pm 0.69$ & 0.73 \\
\hline $\begin{array}{l}\text { Turns-Turn } \\
\text { (degrees/s) }\end{array}$ & $197.81 \pm 40.90$ & $193.64 \pm 42.11$ & 0.59 & $173.47 \pm 22.83$ & $174.72 \pm 43.85$ & 0.93 & $191.39 \pm 35.62$ & $201.61 \pm 38.86$ & 0.60 \\
\hline Turns-Steps in Turn (\#) & $3.94 \pm 0.74$ & $3.91 \pm 0.88$ & 0.58 & $4.51 \pm 0.80$ & $4.42 \pm 0.72$ & 0.77 & $4.33 \pm 0.62$ & $3.85 \pm 0.61$ & 0.15 \\
\hline
\end{tabular}


Table 2 shows the comparisons of gait variables among the different tasks. The results of repeated measures ANOVA are shown in Table 2, with the significance level set at $\mathrm{p} \leq 0.01$. No findings within this table were found to be statistically significant; however, four trends were observed with near significant values.

The first trend observed was gait cycle duration asymmetry (\% Diff) after drinking a Bang ED, which changed from $0.04 \pm 0.32$ to $-0.17 \pm 0.22$, resulting in a p-value of 0.07 . The second noticeable trend observed was lumbar ROM measured in the coronal plane where Rockstar changed from $7.48 \pm 1.48$ to $6.34 \pm 1.37^{\circ}$, resulting in a pvalue of 0.06. Then there was Double support asymmetry which was measured in (\% difference) before and after a Rockstar energy drink and the change was $-0.12 \pm 0.88$ to $0.46 \pm 0.45$ which resulted in a p-value of 0.05 . The final trend that was noteworthy in this table was with Arm ROM on the left side measured in degrees. The change was from $46.36 \pm 8.64$ to $52.03 \pm 4.66$ and the p-value was 0.05 .

Table 3 displays anticipatory postural adjustments among the different tasks. The results of repeated measures ANOVA are shown in Table 3, with the significance level set at $\mathrm{p} \leq 0.05$. No findings within this table were found to be statistically significant; however, one trend can be observed with a near significant value. Anticipatory postural adjustment for forward APA Peak $\left(\mathrm{m} / \mathrm{s}^{2}\right)$ changed from $0.51 \pm 0.34$ pre-Rockstar to $0.87 \pm 0.36$ postRockstar, resulting in a P-value of 0.07.

Table 4 presents comparisons of posture and turns among the different tasks. The results of repeated measures ANOVA are shown in Table 3, with the significance level set at $\mathrm{p} \leq 0.05$. No findings within this table were found to be statistically significant, and no trends were observed. The p-values for all measures in this table are too great to determine any statistically significant or possible trends from consuming an energy drink.

\section{DISCUSSION}

This study aimed to determine the potential impacts on gait following the consumption of an energy drink. The aim was to validate the claims of both energy drinks in general, specifically Redbull, Bang, and Rockstar, that they could improve physical performance. Finally, the data collected rendered no significant findings or alterations in performance following the consumption of energy drinks. However, a couple of trends were discovered that warrant further investigation.

The first main finding of this study revealed that Rockstar or its ingredients might modify gait cycle duration asymmetry, lumbar ROM in the coronal plane, and double support asymmetry compared to the other two drinks. This statement suggests that some of the ingredients of Rockstar, such as guarana, ginseng, and milk thistle, that are not in Redbull or Bang. Guarana has been reported to improve alertness, reaction time, speed of information processing, memory, mood, and physical exercise performance (16). While few studies have validated these claims, sufficient information is available to suggest that it could provide these benefits. Additionally, ginseng has been included in several E Drinks because of its similar effects on the body as guarana, as well as increased effects when interacting with guarana. However, a systematic review and meta-analysis concluded that there is insufficient evidence to provide conclusive indication of the effect of ginseng on physical performance, psychomotor performance, and cognitive function (17). Minimal literature currently exists that supports the use of milk thistle as an additive to $\mathrm{E}$ drinks for improved performance of physical or cognitive tasks, or that justifies the benefits of mixing these three previous ingredients to have a great effect, then each on their own. Although not related to energy drinks, other factors that alter gait are an imbalance in the propulsion of a stronger and weaker limb (22). This implies that any of the above listed ingredients mimics the imbalance of dominant and non-dominant limb propulsion during walking.

Some limitations of this study that apply to this finding are that we were not able to isolate what exactly in Rockstar caused more changes in gait and posture variables compared to Redbull and Bang. This could be due to the widely different active ingredients listed in each of the energy drinks. The main active ingredients present are glucuronolactone and taurine in Redbull, supercreatine in Bang, and the previously listed ingredients for Rockstar. 
Future research should attempt to determine whether Rockstar leads to more changes in posture/gait variables and neuromuscular components. Additionally, further investigation is needed into how these ingredients in isolation affect gait and posture variables ideally at the same dosage that Rockstar deploys these ingredients in their products.

The second main finding of this study revealed gait asymmetry between single-leg stance times after energy drink consumption. These results suggest that E drinks such as Rockstar and Bang could alter the symmetrical pattern of gait by increasing the variable, time, or speed on one side of the body. Patrick and Ingraham (2016) showed that glucose and fructose ingestion can increase stride length during self-paced running (19). In contrast to our study, glucose and fructose concentrations in Rockstar were possibly involved in increasing ROM in the lumbar region or may have led to more asymmetrical movement in general. Additionally, gait asymmetries have been shown between people with a normal BMI and obese BMI. While not akin to our study, they discovered that people with an average body weight tend to have longer stride lengths and have a longer single support line (20). "The single support line was measured by the line of the force application points during the single-support phase" (20). Due to the varying weights of the participants included in the study, the alteration could be due to evidence that body weight can cause a change in gait variables.

This second finding is limited because there was no statistical significance to these trends, which can likely be attributed to chance. In future studies, it would be advantageous to focus on the effects of Rockstar alone on these gait and posture variables to increase the power to detect a statistical difference. Another recommendation would be to have the participants walk longer distances with gait to see if more differences are noted. Ideally, a greater amount of time should be spent with each subject per trial to elicit the effects of caffeine since research states that the effects of caffeine in the body are felt about 20 minutes after ingestion (21).

\section{CONCLUSION}

The intention of this study was to determine the effects of different types of energy drinks on gait parameters. Specifically, the goal was to determine whether Rockstar, Bang, or Redbull had a larger change in gait parameters including but not limited to elevation at midswing asymmetry, gait cycle duration right and left, and gait speed right and left. There were no significant findings in this study, although it was shown that Rockstar may lead to more gait deviations such as modifying gait cycle duration asymmetry, lumbar ROM in the coronal plane, and double support asymmetry compared to the other two drinks. We conjecture that this might be due to the different ingredients, such as guarana, milk thistle, and ginseng, or the interactions between these ingredients and caffeine. Additionally, as proposed by Rosario et al. (2017) there is a possibility that some of the ingredients in these E drinks are causing neuromuscular modifications in lower limb muscles, thus making this remark a future direction for the current study. The contribution of this research to the literature is that Redbull should be investigated further to determine if the drink does in fact cause significant differences in gait. It also shows that energy drinks have a possible link to gait deviations. Future research should focus on the effects of Rockstar on more subjects to gain more statistical power as well as using a different variety of subjects with different ages to see if it affects different ages differently. The simplest suggestion for future research in this area is to only have the subjects walk for longer periods of time while collecting neuromuscular data to understand dynamic postural control while walking.

Funding: This study received financial support from Texas Woman's University Small Grant Program.

Competing Interests: The authors declare that they have no competing interests.

Acknowledgement: All authors contributed equally to the conception and design of the study. 


\section{REFERENCES}

Grgic, J., Trexler, E. T., Lazinica, B., \& Pedisic, Z. (2018). Effects of caffeine intake on muscle strength and power: A systematic review and meta-analysis. Journal of the International Society of Sports Nutrition, 15(1), 1-10. Available at: https://doi.org/10.1186/s12970-018-02 16-0.

McNerney, K. M., Coad, M. L., \& Burkard, R. F. (2014). The influence of caffeine on the sensory organization test. Journal of the American Academy of Audiology, 25(6), 52 1-528. Available at: https://doi.org/10.3766/jaaa.25.6.2.

Patrick, W. B., \& Ingraham, S. J. (2016). Effects of glucose-fructose versus glucose ingestion on stride characteristics during prolonged treadmill running. Sports Biomechanics, 15(3), 270-282. Available at: https://doi.org/10.1080/14763141.2016.1159726.

Rosario, M. G., Collazo, H., Mateo, M., Gonzalez-Sola, M., \& Bayron, F. (2017). Increased static postural sway after energy drink consumption: A randomized trial [version 1; peer review: 2 approved with reservations]. F100oResearch 2017, 6, 2036. Available at: https://doi.org/10.12688/f1000research.12565.1.

Rosario, M. G., Clare, S. H., Lauren, S. B., \& Ashley, S. C. (2021). Ankle complex musculature adaptations after Energy drinks consumption in healthy young adult. International Journal of Physical Education, Fitness and Sports, 10(2), 32-44. Available at: https://doi.org/10.34256/IJPEFS2124.

Views and opinions expressed in this article are the views and opinions of the author(s), Journal of Sports Research shall not be responsible or answerable for any loss, damage or liability etc. caused in relation to/arising out of the use of the content. 